\title{
PERFIL DE LOS PARTICIPANTES DE INTERNADOS DE MAPUZUGUN DE LA ORGANIZACIÓN MAPUZUGULETUAIÑ
}

\section{PROFILE OF PARTICIPANTS IN MAPUCHE LANGUAGE IMMERSION PROGRAMS ORGANISED BY MAPUZUGULETUAIÑ}

\author{
Maria-Jesus Inostroza Araos \\ Universidad de Concepción - Mapuzuguletuaiñ, Wallmapu \\ minostrozaa@udec.cl \\ Ester Quero Álvarez \\ Instituto Nacional de la Lengua Mapuche, Mapuzuguletuaiñ, Wallmapu \\ ester.quero.a@gmail.com \\ Aldo Berríos Castillo \\ Universidad de Concepción - Mapuzuguletuaiñ, Wallmapu \\ aldoberrios@udec.cl
}

\begin{abstract}
Resumen:
En la última década, el creciente interés por aprender la lengua mapuche ha aumentado fuertemente, siendo organizaciones autónomas las que responden a esas necesidades. Sin embargo, poco se sabe acerca de los participantes de las instancias de aprendizaje. El propósito de este artículo es indagar en el perfil de las y los estudiantes de dos internados lingüísticos realizados durante el verano de 2020 por la organización Mapuzuguletuaiñ, Instituto Nacional de la Lengua Mapuche. Para ello se usó un cuestionario que permitió describir un perfil del estudiante, según territorio de procedencia, edad, nivel educativo y trayectorias de participación. Este perfil se caracteriza como adultos con estudios universitarios completos, que se auto-identifican de nacionalidad mapuche y que muestran una trayectoria de participación constante y reiterativa dentro de una misma organización.
\end{abstract}

Palabras clave: revitalización, mapuzugun, internado, estudiante, mapuche

\begin{abstract}
:
The last decade has witnessed a growing interest in learning Mapuzugun, the Mapuche language. This need has been addressed by autonomous organizations that manage language immersion programmes. Little is known about who takes part in these learning experiences. This article aims at investigating the profile of the participants in two language immersion courses during the summer of 2020 organized by Mapuzuguletuaiñ, the National Institute of the Mapuche language. A questionnaire was applied in order to identify the place of origin, age, educational level and participation backgrounds. Results show that most of the participants are adults with completed university degrees, who self-identify as Mapuche nationals, and who show a constant and recurrent participation in the same organization.
\end{abstract}

Key words: revitalization, mapuzugun, immersion, student, mapuche 
Artículo. María-Jesús Inostroza, Ester Quero, Aldo Berríos. "Perfil de los participantes de

Recibido: 28 de mayo de 2021

Aceptado: 22 de junio de 2021

\section{Introducción}

El presente artículo busca describir el perfil de las personas que asisten a internados lingüísticos de mapuzugun $^{1}$ organizados por Mapuzuguletuaiñ, Instituto Nacional de la Lengua Mapuche, dedicado a la promoción y enseñanza del mapuzugun en el territorio mapuche, Wallmapu.

El interés por aprender la lengua mapuche ha aumentado en las últimas décadas en concordancia con el aumento de las demandas identitarias del pueblo mapuche (Mayo y Castillo 15), el incremento de la matrícula universitaria de estudiantes mapuche (Zuñiga y Olate 366) y el surgimiento de espacios de revitalización lingüística gestionados por actores sociales (Olate et al. 48). Por otro lado, la transmisión del mapuzugun de manera intergeneracional se ha visto interrumpida debido a las experiencias negativas sufridas por una generación de hablantes que tuvo que enfrentarse a procesos de colonización que implicaron, entre otras cosas, una escolarización que desvalorizaba y castigaba el uso de la lengua mapuche (Antimil y Olate 117).

Asimismo, el poco valor de uso en el contexto urbano de la lengua mapuche también llevó a la decisión de algunas personas a dejar de hablarlo. En este contexto han surgido experiencias de transmisión no tradicional, tales como talleres e internados lingüísticos que buscan aumentar el número de hablantes y promover el uso del mapuzugun (Zuñiga y Olate 365, Olate et al. 48). Estas iniciativas se caracterizan por ser concretadas por la sociedad civil a través de organizaciones denominadas txokiñche o grupos de personas dedicadas a la promoción del mapuzugun como segunda lengua entre jóvenes y adultos mapuche y no mapuche.

Cada año, txokiñche como Mapuzuguletuaiñ dedican tiempo y recursos a la preparación, ejecución y evaluación de estas experiencias. La mantención de este tipo de instancias en el tiempo demuestra el carácter significativo de estas para sus participantes. Sin embargo, a pesar de que estas instancias han sido identificadas en la literatura (Mayo y

\footnotetext{
${ }^{1}$ Las palabras en mapuzugun que aparecen en este artículo se escriben en el alfabeto Azümchefe, que se caracteriza por usar $\langle\mathrm{z}\rangle,\langle\mathrm{tx}\rangle,\langle\mathrm{g}\rangle$ y $\langle\mathrm{q}\rangle$ en lugar de $\langle\mathrm{d}\rangle,\langle\mathrm{tr}\rangle,\langle\mathrm{ng}\rangle, \mathrm{y}\langle\mathrm{g}\rangle$ del Unificado. Una descripción más acabada de este grafemario se puede consultar en la Gramática básica de la lengua mapuche de Hernández et al (36-38) o en Azümchefe de CONADI.
} 
Castillo 15, Olate et al 48) como relevantes en el proceso de revitalización de la lengua, poco se sabe acerca de las personas que participan en ellas.

Conocer el perfil de las personas que asisten a estas instancias tiene implicancias en las decisiones pedagógicas que se adopten antes y durante la ejecución de estas experiencias de revitalización, además de las disposiciones logísticas respecto de la inversión de recursos. El estudio de caso de estudiantes que son parte de esta iniciativa permitirá conocer sus perfiles demográficos, además de su participación en general en instancias de aprendizaje del mapuzugun.

Para entender quiénes están detrás de las experiencias de inmersión de los internados lingüísticos es necesario comprender el rol histórico que ha tenido el idioma mapuche y las circunstancias que hicieron que perdiera ese estatus en el territorio denominado Wallmapu, actualmente ocupado por los Estados chileno y argentino.

\section{Marco teórico y conceptual}

\subsection{El contexto histórico del mapuzugun}

El territorio mapuche contiene los asentamientos humanos más antiguos del continente americano (Moreno et al 2). Históricamente, el mapuzugun se hablaba en una gran extensión territorial desde el río Aconcagua hasta la isla de Chiloé (Adelaar 502). Se sabe que el mapuzugun estuvo en contacto con otras lenguas, como queda demostrado por una serie de préstamos lingüísticos incorporados al mapuzugun durante el desarrollo del imperio Inca en el siglo XV (Moulian et al 268). Asimismo, es sabido que fue hablado como segunda lengua por otros pueblos vecinos, según quedó registrado por los cronistas de la época (Ovalle 101).

Desde la llegada del conquistador español, el mapuzugun fortaleció su uso comunicativo, pero ahora como instrumento para la conquista y evangelización por parte de misioneros jesuítas (Gaune 15), algunos de los cuales lo hablaban de manera natural al haber nacido en este territorio o bien lo aprendían como segunda lengua. Igualmente, se notó su uso como instrumento de comunicación en la naciente economía colonial de la época (Amberga 420). 
Artículo. María-Jesús Inostroza, Ester Quero, Aldo Berríos. "Perfil de los participantes de internados de mapuzugun de la organización Mapuzuguletuaiñ"

Esta necesidad comunicativa para el proyecto evangelizador del mapuzugun llevó al establecimiento de cátedras para aprender el idioma mapuche. En 1697, por ejemplo, se estableció una clase de lengua mapuche para los misioneros en la ciudad de Concepción (Amberga 423). Así el mapuzugun se convirtió en una lengua registrada en la escritura desde temprana época según se evidencia por las gramáticas de Luis de Valdivia de 1606 y, posteriormente, por los trabajos de Havestadt en 1765 y Febres en 1777, todos en el contexto de evangelización.

Ya establecida la república de Chile, y motivado por una crisis económica mundial, entre otros factores, los Estados chileno y argentino comienzan una invasión militar al territorio mapuche, además de una incorporación del pueblo mapuche a la sociedad nacional (Cisternas y Vallejos 129). Con estos hechos sangrientos se da inicio a una era de violencia material y simbólica ejercida desde el aparataje estatal que se traduce en la prohibición de cualquier manifestación de la cultura propia mapuche, lo que afectó especialmente al mapuzugun (Cisternas 4) y propició el aprendizaje y transmisión de la lengua dominante entre la población mapuche (Canales 28).

A partir de los procesos de ocupación del territorio mapuche, mal denominados "Pacificación de la Araucanía" en Chile y "Conquista del Desierto" en Argentina, el mapuzugun comenzó a ser desplazado como lengua general y reducir sus funciones de uso, concentrándose en el dominio comunitario y familiar. Con la profundización de los procesos históricos de ocupación y despojo material, se produjo un éxodo desde las comunidades mapuche hacia las periferias urbanas, lo que se tradujo en el abandono del mapuzugun y el quiebre de la transmisión intergeneracional. Asimismo, dentro de las comunidades se comenzaron a asentar los agentes y dispositivos civilizatorios, espacios tales como la iglesia y la escuela, que propiciaron el desplazamiento del mapuzugun (Antimil y Olate 18).

En respuesta a esta apremiante situación, en la primera década del siglo XX, surge el movimiento mapuche moderno. Tres hechos marcan el inicio de este periodo. En 1910, se funda la primera organización mapuche, la Sociedad Caupolićan Defensora de la Araucanía (Naguil 40). En 1913, en víspera del año nuevo mapuche, José Manuel Painemal fue marcado por un hierro incandescente cerca de Nueva Imperial. Este hecho fue denunciado por la Sociedad Caupolicán y generó una concentración en esta ciudad el 6 de julio de 1913 con más de 3000 asistentes mapuche (Pinto 15). Con estos hechos se inicia un periodo nuevo en 
las demandas por la identidad mapuche y acciones de resistencia frente a los hechos devastadores del proceso colonialista que sufre la sociedad mapuche.

\subsection{Estado actual de vitalidad del mapuzugun}

Actualmente, la situación del mapuzugun es diversa y no estabilizada. De acuerdo con UNESCO se trata de una lengua en peligro, lo que implica que "los niños ya no la aprenden en sus familias como lengua materna" (Moseley 81). Conjuntamente, la mayoría de los estudios de vitalidad caracterizan al mapuzugun como una "lengua minoritaria y minorizada" (Zuñiga y Olate 368), de vitalidad y viabilidad "severamente amenazadas" (Zuñiga 23), de "estado recesivo" (Gundermann et al., Permanencia 56), en un "escenario de retroceso funcional" (Lagos 181) y en "proceso de desplazamiento" (Wittig e Israel 124). En lo que respecta al uso del mapuzugun en la ciudad es evidente la inexistencia práctica del mapuzugun con valor de uso.

La situación antes descrita radica en procesos de colonización chilena de fines del siglo XIX "donde los colonizadores traen un sistema de comunicación distinto que debe ser incorporado, obligatoriamente, en las dinámicas comunicativas inter-étnicas y, prontamente, intra- étnicas." (Antimil y Olate 119). De esta forma, se establecen políticas educativas donde se penaliza el uso de la lengua (Ñanculef y Cayupan 29) y se instaura el aprendizaje del castellano como parte de un proceso de incorporación cultural e institucional a la sociedad chilena (Catrileo 38). Esta "minorización y absorción cultural" (Antimil y Olate 120) se ve fuertemente marcada en el contexto urbano (Salas 29).

El proceso de migración mapuche a la ciudad se inició en la década del 1930 (Canales 4); sin embargo, este se amplió fuertemente desde la década de 1960. Las ciudades que fueron centros de radicaciones urbana mapuche son Temuco, Concepción y Santiago (Gunderman et al. Permanencia 55). Producto de la migración hacia los polos urbanos mencionados, se produce la emergencia de un espacio no tradicional, donde la transmisión del idioma ya no es dentro de un contexto familiar entre mayores y niños, sino que en talleres de lengua entre adultos y con medios más "artificiales” de reproducción (Lagos 181). Es así como comenzaron a surgir movimientos de revitalización a cargo de agrupaciones, colectivos y organizaciones que trabajan en la enseñanza y aprendizaje del mapuzugun (Mayo y Castillo 
Artículo. María-Jesús Inostroza, Ester Quero, Aldo Berríos. "Perfil de los participantes de internados de mapuzugun de la organización Mapuzuguletuaiñ"

15). El trabajo de estas organizaciones se proyecta hasta el día de hoy, por lo que se puede hablar de un proceso histórico de resistencia ante las acciones asimilatorias del Estado chileno.

En este contexto se desarrolla la emergencia del hablante urbano y los nuevos agentes de transmisión, además de espacios de socialización no tradicionales. Surgen nuevos espacios de interacción y transmisión, como talleres informales, donde se produce una reproducción del idioma que se contrapone a la transmisión intergeneracional. Dentro de este contexto, surge la figura del aprendiz del idioma, conceptualizado en mapuzugun con el vocablo de chillkatufe (palabra que tiene varios significados: lector, colegial, estudiante) quien participa de instancias de aprendizaje caracterizadas por su autonomía y espontaneidad (Lagos 167).

En las últimas décadas, se evidencian fenómenos de resistencia cultural asociadas a la lengua, como movilizaciones y actividades en torno a la reivindicación por la oficialización y la revitalización del mapuzugun (Naqill 136). Estas han sido convocadas e impulsadas por organizaciones donde participa gente joven, muchos de ellos estudiantes secundarios y universitarios (Naqill 139).

En este contexto surgen iniciativas de inmersión lingüística, especialmente de organizaciones que desarrollan espacios de educación autónomos (Mayo y Castillo 15, Loncón et al. 199). La experiencia de "internado lingüístico", traducido y adaptado en mapuzugun por el concepto de koneltun mapuzugun mew ${ }^{2}$, son instancias de enseñanza y aprendizaje del mapuzugun mediante inmersión lingüística con una duración de varios días y que se realizan principalmente durante verano o vacaciones de invierno (Olate et al. 52, Naqill 138). En uno de los pocos artículos académicos escritos íntegramente en mapuzugun, Elisa Loncon describe estas experiencias como "wechekeche ñi küzaw, magelkefi küpa mapuzugulu, txawün mu chillkatukeygün, kimkantukey, ülkantukey, konpamapuzugukeygün ${ }^{3 "}$ (Mapuzugun 220).

\footnotetext{
${ }^{2}$ La expresión koneltun mapuzugun mew significa "entrar, meterse o participar en el mapuzugun". Si bien koneltun es un verbo, en mapuzugun estos se pueden nominalizar; es decir, también funcionan como sustantivos que nombran la acción de ese verbo.

${ }^{3}$ Este artículo cuenta con una traducción en castellano que reproducimos aquí: "Es el trabajo de los jóvenes que invitan a quienes desean aprender el mapuzugun, en grupo y en el contexto de la comunidad lo enseñan, lo estudian, lo cantan, retoman el uso de la lengua." (Loncon El mapuzugun 217).
} 
Un componente siempre presente en estas experiencias de aprendizaje es la territorialización. En palabras de Tomás et al., estas estrategias se acompañan “de otras recuperaciones, como la del territorio" (42). Para entender esta dimensión es importante precisar que existen dos concepciones de lo que sería el territorio nacional mapuche, por un lado el Wallmapu "independiente" de menores dimensiones, y que comprende la región de La Araucania junto con comunas adyacentes de las regiones del Bío-Bío y de los Ríos) versus

un Wallmapu que involucra una mayor área (Río Bío-Bío hasta Chillwe en su eje longitudinal, además de las zonas cordilleranas de las provincias argentinas de Neuquén, Río Negro y Chubut) (Naquil 151).

\subsection{Identidad y el aprendizaje de idioma}

La recuperación de los espacios de aprendizaje del mapuzugun fuera de los entornos familiares se muestra como una respuesta a la necesidad de reconectar el manejo del idioma y el ser mapuche. Esta situación refleja la motivación que existe respecto a aprender mapuzugun. Ajello (2003) señala que la motivación debe ser entendida como la trama que sostiene el desarrollo de aquellas actividades que son significativas para la persona y en las que esta toma parte. En el plano educativo, la motivación debe ser considerada como la disposición positiva para aprender y continuar haciéndolo de una forma autónoma (252).

En particular, en lo que refiere a la motivación por aprender un idioma, el constructo de la orientación integradora correspondiente a la teoría de motivación de Gardner se ha mencionado como aplicable al contexto de idiomas en revitalización. La orientación integradora se define como "la motivación por aprender una segunda lengua debido a los sentimientos positivos hacia la comunidad que habla esa lengua" (Gardner 82-83). La integratividad refleja "un interés genuino por aprender la segunda lengua para acercarse a la otra comunidad lingüística" (Gardner 5). Sin embargo, en el caso de los aprendices de mapuzugun, u otras lenguas de herencia, no se busca "intentar acceder a otra cultura (extranjera), sino a la suya propia, que a menudo se ha perdido para ellos, su familia o su comunidad" (McIvor 88). Respecto de las implicancias del aprendizaje de lenguas de herencia por parte de generaciones más jóvenes que pertenecen a dichos pueblos, hay estudios que muestran que a través del proceso de aprendizaje estos aprendices se sienten 
conectados con su comunidad y se identifican con su historia, cultura y las generaciones mayores (Moore 4, Nicholas 361). En el caso particular del mapuzugun, en las palabras de Lagos, "se la sigue considerando un elemento nuclear en la definición de lo que significa ser mapuche en la actualidad" (181), configurándose como un aspecto relevante de la reafirmación de identidad (Llanquinao et al 125).

Es así como se identifican dos perspectivas teórico/pedagógicas que guían los procesos de enseñanza-aprendizaje de la lengua, mientras una de ellas está "basada en las epistemologías y metodologías propias de los hablantes" (Llanquinao et al 124), la otra responde a el "método multidimensional con enfoque comunicativo de enseñanzaaprendizaje de la lengua mapuche, como segunda lengua" (Becerra 96) altamente informada

por las experiencias de aprendizajes de idiomas a nivel internacional. La primera perspectiva, argumenta la necesidad de respetar las formas propias de la transmisión lingüística y cultural de la lengua focalizada en la oralidad y intergeneracionalidad con el fin de ser replicada en contextos de educación formal como son la escuela (Llanquinao et al 127). Por otro lado, la aproximación a la enseñanza del mapuzugun a través del método comunicativo considera el conocimiento generado del proceso de aprendizaje de una segunda lengua de otros idiomas, al mismo tiempo que destaca "la riqueza del zugu y entre otros la potencia de la tradición oral del mapuzugun" (Namuncura 62).

\section{El estudio}

El presente estudio tiene una aproximación cualitativa y de naturaleza exploratoria, correspondiendo a un estudio intrínseco de caso (Stake 16). Dicho caso se conformó por las personas que asistieron a koneltun de mapuzugun organizados por el txokiñche Mapuzuguletuaiñ. Los objetivos definidos son: (a) describir el perfil de los asistentes a koneltun de mapuzugun organizados por un txokiñche, y (b) dar cuenta de la trayectoria participativa de aprendizaje de los chillkatufe del mapuzugun como segunda lengua antes del internado lingüístico.

Para el logro de los objetivos presentados se trabajó con una muestra selectiva compuesta por 85 participantes de dos internados lingüísticos de mapuzugun realizados en Panguipulli, Región de Los Ríos, y Tirúa, Región del Bío-Bío, Chile. Estos internados se realizaron en temporada estival (walüg koneltun, en mapuzugun), específicamente del 19 al 
24 de enero de 2020 para el caso del internado de Panguipulli y del 17 al 25 de febrero de 2020, en el caso del internado de Tirúa. Cabe señalar que durante esta temporada se realizaron otros dos internados, tanto en Osorno como en las proximidades de Angol; sin embargo, estos no forman parte del presente estudio.

Para la recolección de datos se diseñó y aplicó una encuesta que consideraba edad, lugar de procedencia, nivel de escolaridad, ocupación, nacionalidad y participación previa en cursos y/o koneltun. Dichos datos fueron analizados a través de estadísticas descriptivas y análisis de frecuencia.

\subsection{Mapuzuguletuaiñ}

El txokiñche Mapuzuguletuaiñ o Mapuzuguletuaiñ Wallmapu mew ("Volveremos a hablar mapuzugun en Wallmapu”) es una instancia de revitalización lingüística surgida desde la sociedad civil. Este txokiñche es una organización compuesta por personas mapuches y no mapuches que se dedica a la revitalización lingüística del mapuzugun, a través de su enseñanza, promoción y preservación, y dirigida a las personas habitantes de Wallmapu.

En septiembre de 2017, este txokiñche se constituye como corporación bajo la personalidad jurídica "Instituto Nacional de la Lengua Mapuche Mapuzuguletuaiñn". No obstante, antes de su constitución, ya había realizado actividades de koneltun mapuzugun mew en el verano de 2017 (Quidico, Reigolil, Temuco), además de un internado en Puel тари (Zapala), donde se hizo cargo de la pedagogía. Esto marcó un inicio en la colaboración con organizaciones trasandinas.

En base a una necesidad no satisfecha, esto es, la falta de espacios de enseñanza y promoción del mapuzugun, este txokiñche dirige sus acciones hacia la toma de decisiones propias como población afectada. De esta manera, el grado de participación juega un rol fundamental para su sustentabilidad en el tiempo, ya que las acciones son pensadas por la población y compartidas por agentes externos de desarrollo (Arnstein 32) y no viceversa. Un ejemplo claro de esto es la vinculación con la asociación Garabide, ONG de Euskal Herria (país vasco) abocada a apoyar procesos de revitalización lingüística de lenguas minorizadas a partir de la experiencia del euskera (Garabide). Esta vinculación se ha llevado a cabo por 
medio de pasantías y capacitaciones en el área técnico-pedagógica de la enseñanza de idiomas (Naqill 138).

En término de las estrategias de enseñanza y aprendizaje definidas por el txokiñche, en un principio se adoptó un enfoque basado en la metodología de la gramática-traducción. Sin embargo, por considerarse un enfoque obsoleto (Namuncura 63) y en vista de los resultados, la organización avanzó hacia el enfoque comunicativo. De esta manera, la organización demuestra ser reflexiva en torno a la búsqueda de estrategias eficientes para lograr niveles óptimos de la lengua. El Enfoque Comunicativo como estrategia de enseñanza del idioma "hace hincapié en la autenticidad, la interacción, el aprendizaje centrado en el estudiante, las actividades basadas en tareas y la comunicación para el mundo real, con fines significativos (Brown y Lee 629). Dicho enfoque se articula con el Marco Común de Referencia para las Lenguas (Namuncura 64), el que define competencias lingüísticas en escalas de niveles de acuerdo a horas de aprendizaje desde un A1, nivel inicial de la lengua, hasta un $\mathrm{C} 2$, para aquellos que dominan el idioma de manera competente.

Mapuzuguletuaiñ se caracteriza por dirigir su estrategia de revitalización dentro de un contexto territorial delimitado (Naqill 145), lo que explica que sus cursos e internados estén orientados a todos los habitantes del territorio, sean estos o no mapuche. En palabras de Mapuzuguletuaiñ, "el mapuzugun debe tener un uso cotidiano en todos los espacios en que nos desenvolvemos como mapuche y habitantes del territorio" ("Mapuzuguletuaiñ”). Ejemplo de lo anterior es que existe presencia de personas del territorio ocupado por el Estado de Argentina, denominado Puel mapu (Tierra del Oriente), en las actividades que realiza Mapuzuguletuaiñ en Gulu mapu (Tierra de Occidente, o el territorio ocupado por el Estado de Chile). Asimismo, Mapuzuguletuaiñ realiza actividades de Koneltun mapuzugun mew en Puel mapu en conjunto con organizaciones trasandinas desde el año 2018, haciéndose cargo de los aspectos pedagógicos (Tomás et al. 40).

Para Mapuzuguletuaiñ, la revitalización del mapuzugun es un proceso de reconstrucción nacional. El mapuzugun es la lengua propia de una comunidad humana, el pueblo mapuche, y es también la lengua propia de un país, Wallmapu (Naqill 150). 


\section{Resultados}

\subsection{Perfil demográfico de los asistentes a koneltun}

\subsubsection{Población}

En términos absolutos, se evidencia la asistencia de adolescentes, adultos y adultos mayores en internados lingüísticos. A su vez, es posible identificar que en ámbitos etarios existe una media de 29,43, una moda de 28 , una desviación típica de 8,98 y una varianza de 80,64 (véase Figura 1). Dichas estadísticas permiten comprender que existe una gran dispersión en ámbitos etarios; no obstante, a su vez permite identificar que la participación mayoritaria de pu chillkatufe asistentes en dichos espacios de aprendizaje se encuentran en un periodo de ciclo vital de adultez temprana ${ }^{4}$, seguido por adultez intermedia ${ }^{5}$. Este grupo es coherente con la descripción identificada en la literatura, donde existe un grupo de adultos que no recibió la transmisión del idioma de padres o familiares que buscan aprender el idioma en contextos no tradicionales (Lagos 181). Asimismo, es posible observar una prevalencia de chillkatufe entre 21 y 40, lo que se condice con los datos de vitalidad del 2007 que muestran que la mayoría de las personas mapuche entre 10 y 29 años no tenían competencias en el idioma (Gundermann et al 42).

\footnotetext{
${ }^{4}$ Etapas según modelo empírico, desde los 17 a los 44 años de edad, determinada social y psicológicamente como el momento de materializar ilusiones, deseos, satisfacciones personales y selección de estilos de vida.

${ }^{5}$ Etapas según modelo empírico, desde los 45 a los 65 años de edad, caracterizada social y psicológicamente por generación dominante (liderazgo, niveles políticos, religiosos, industriales, científicos y artísticos).
} 
Artículo. María-Jesús Inostroza, Ester Quero, Aldo Berríos. "Perfil de los participantes de internados de mapuzugun de la organización Mapuzuguletuaiñ"

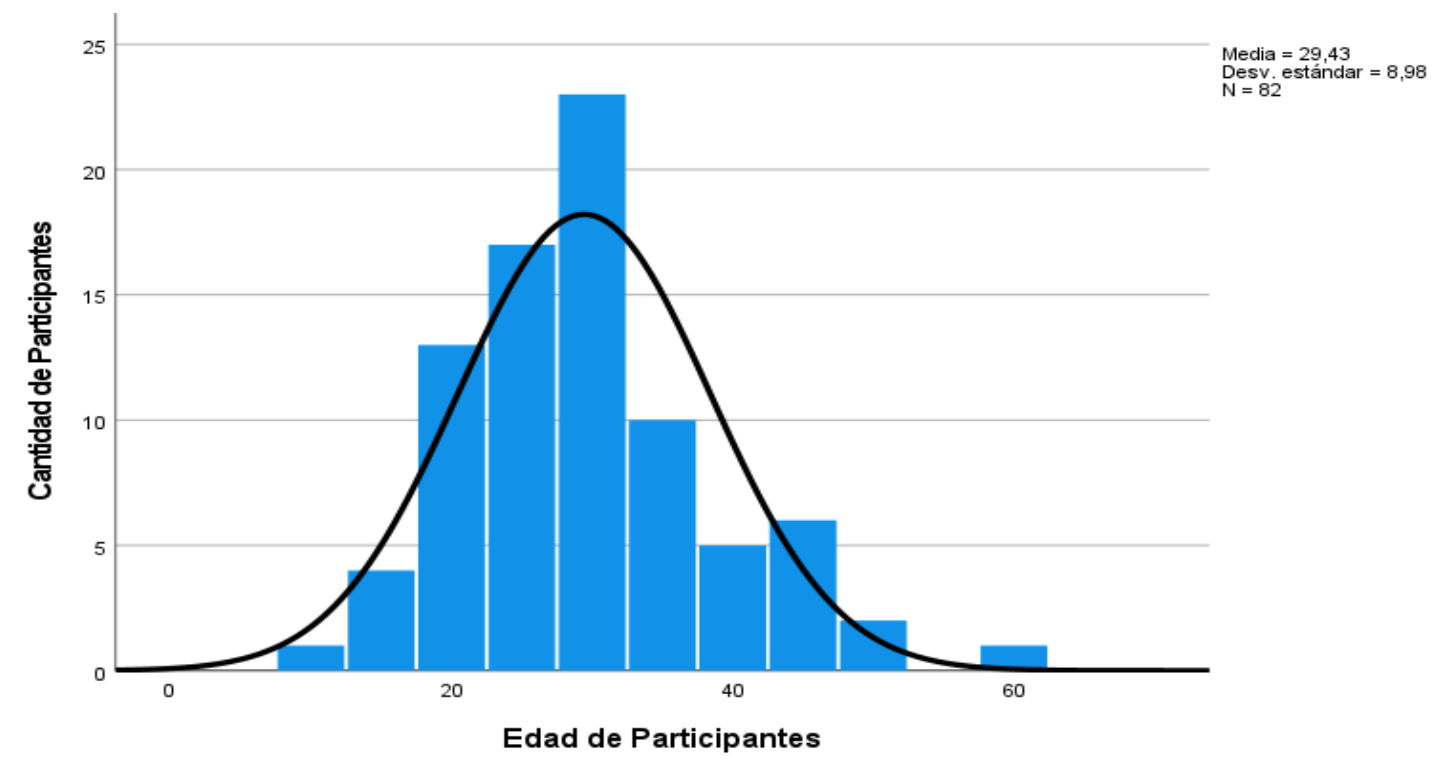

Figura 1. Distribución etárea de pu chillkatufe

En relación a la afiliación e incorporación de hombres y mujeres ante este tipo de espacios educativos, se presenta una prevalencia mayoritaria alcanzada por mujeres, siendo estas representantes del 69,51\% de la población total que asistió a dichos koneltun, mientras que el 30,48\% restante responde a la asistencia de hombres. De esta manera, si comparamos la relación participativa de hombres y mujeres y su rango etario, son las mujeres entre los 21 a 30 años quienes más asistieron a los respectivos internados, mientras que la tendencia mayoritaria de hombres fue sobre los 26 años. 


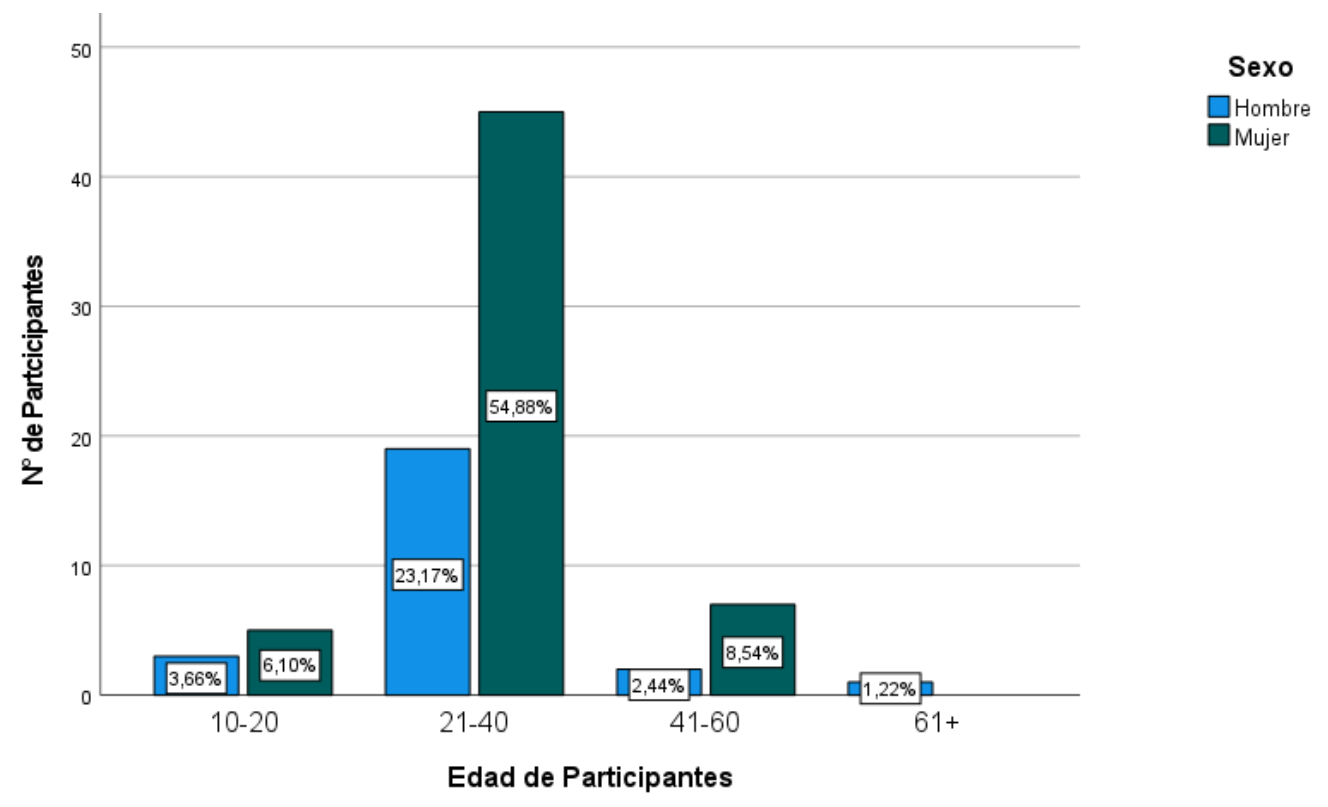

Figura 2. Distribución porcentual según edad y sexo de pu chillkatufe

\subsubsection{Ocupación y escolaridad de la población}

En lo que respecta a la distribución de pu chillkatufe asociada al nivel de escolaridad y ocupación, se identificó de manera descendente el siguiente prospecto: un 37,98\% trabajaba, situación que permite identificar actividades laborales remuneradas sean estas formales o informales como ocupación. En segunda instancia un 25,34\% seleccionó como ocupación el estudiar, aspecto relacionado a ámbitos formales de educación. En tercera instancia un 25,32\% manifestó trabajar y estudiar, mientras que sólo un 11,4\% de los encuestados dio a conocer que se encontraban desocupados, es decir, sin participación formal remunerada o académica formal. 
Artículo. María-Jesús Inostroza, Ester Quero, Aldo Berríos. "Perfil de los participantes de internados de mapuzugun de la organización Mapuzuguletuaiñ๊"

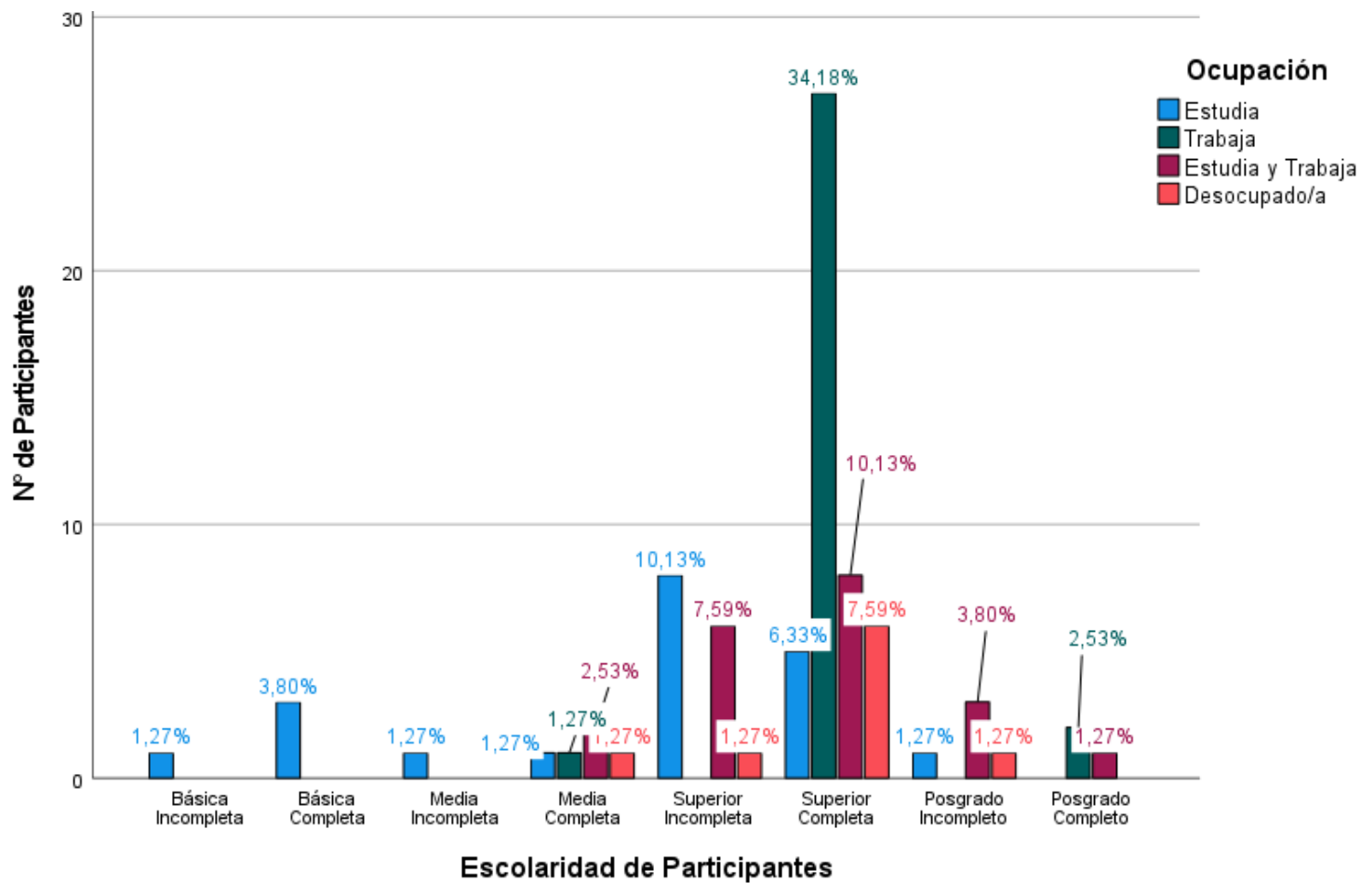

Figura 3. Distribución porcentual según ocupación y escolaridad de pu chillkatufe

Por tanto, existe una prevalencia participativa de pu chillkatufe asistentes que cuentan con estudios formales en educación superior, representando un $87,36 \%$ del total de la población, donde un 58,23\% declara haber cursado educación superior completa en calidad de egresados o titulados y un 18,99\% declara un nivel de educación superior incompleta, un $6,34 \%$ posgrado incompleto y un $3,8 \%$ posgrado completo. Este factor da cuenta de las estrategias desarrolladas por la organización, las que tienden a convocar a personas participantes de espacios de educación formal superior. Este alto número de participantes con estudios superiores se relaciona o es coherente con el aumento de matrículas de estudiantes mapuche en instituciones de estudios superiores (Zuñiga y Olate 365) y a la historia reciente del movimiento por la revitalización donde han participado organizaciones estudiantiles (Mayo y Castillo 15, Naqill 139).

En relación al nivel educativo y edad se deja en evidencia que el rango etario con mayor participación en dichos koneltun se encuentra entre los 21 a 40 años, representando un $79,75 \%$ del total de la población, donde la mayor prevalencia en relación al nivel educativo oscila entre educación superior completa y educación superior incompleta. 


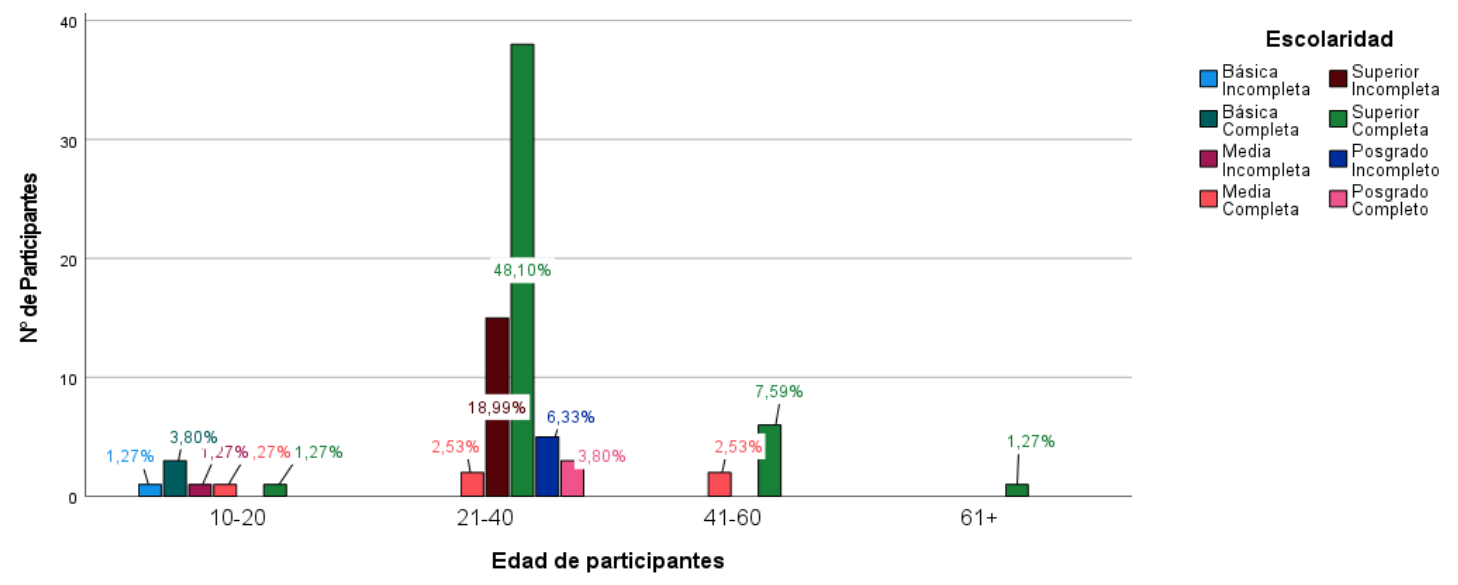

Figura 4. Distribución porcentual según Escolaridad y Edad de pu Chillkatufe

\subsubsection{Lugar de procedencia según región de la población}

La participación de pu chillkatufe en los koneltun de mapuzugun realizados en Panguipulli y Tirúa, tenían como lugar de procedencia 7 regiones de Chile, entre las cuales encontramos: R. de Valparaíso, R. Bernardo O’Higgins, R. del Bío-Bío, R. de los lagos, R. Metropolitana, R. de los ríos y la R. de la Araucanía. En necesario además considerar que en esta muestra de estudio también participaron pu chilkatufe de Argentina, motivo por el cual situaremos Patagonia como Región, ya que en el instrumento se explicitan dos provincias del sector: Neuquén y Río Negro. En relación a los lugares de procedencia, un 61,8\% del total de la población proviene de sectores urbanos y un 38,2\% de sectores rurales.

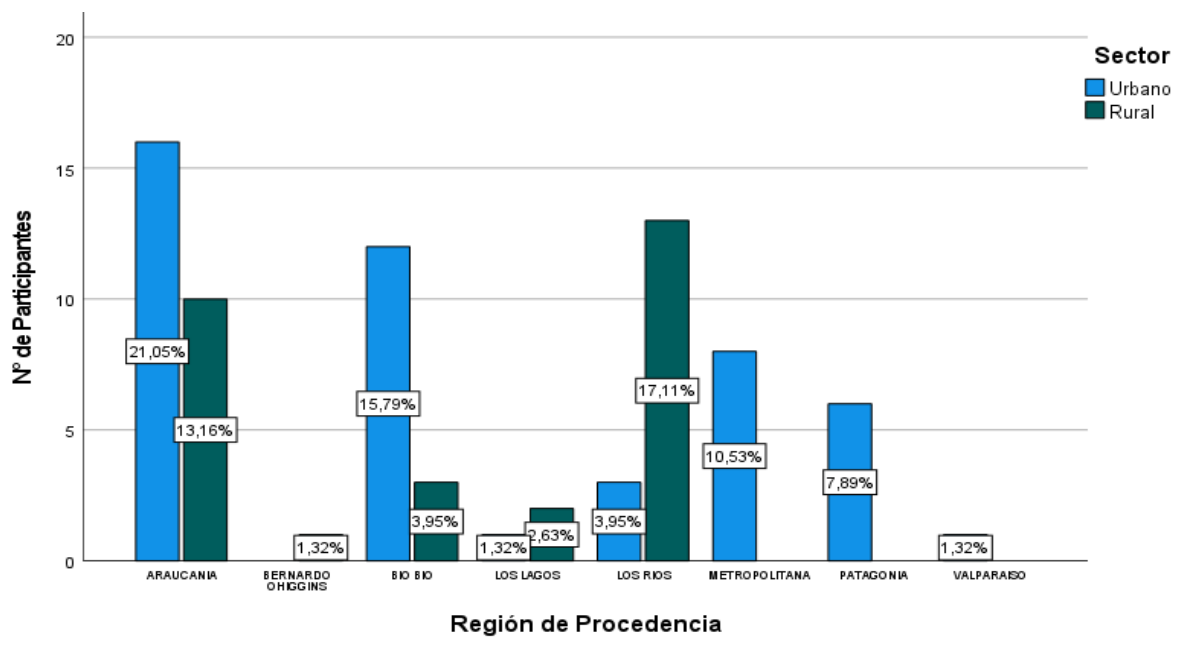

Figura 5. Distribución porcentual según región y sector de pu chillkatufe 
Se puede apreciar que en la muestra existe una prevalencia mayor de procedencia de tres regiones, entre las cuales encontramos la Región de la Araucanía, la Región de Los Ríos y la Región del Bío-Bío, territorios donde en años anteriores se han realizado internados de mapuzugun.

En relación a variables de rango etario asociadas a regiones de procedencia de $p u$ chillkatufe asistentes a koneltun, se observa una mayor prevalencia en Araucanía, Los Ríos y Bío-Bío, representando el 75,61\% del total de la población participante en dichos espacios educativos de aprendizaje.

\begin{tabular}{|c|c|c|c|c|c|c|}
\hline \multirow{3}{*}{\begin{tabular}{|l|} 
Porcentajes \\
Región \\
\end{tabular}} & \multirow[b]{2}{*}{ Región -Edad } & \multicolumn{4}{|c|}{ Edad de Participantes } & \multirow[b]{2}{*}{ Total } \\
\hline & & $10-20$ & 21.40 & $41-60$ & $61+$ & \\
\hline & ARAUCANIA & $3,66 \%$ & $23,17 \%$ & $4,88 \%$ & $1,22 \%$ & $32,93 \%$ \\
\hline \multirow{7}{*}{ Región } & LOS RIOS & $0,00 \%$ & $20,73 \%$ & $2,44 \%$ & $0,00 \%$ & $23,17 \%$ \\
\hline & $\mathrm{BIO} \mathrm{BIO}$ & $2,44 \%$ & $14,63 \%$ & $2,44 \%$ & $0,00 \%$ & $19,51 \%$ \\
\hline & METROPOLITANA & $1,22 \%$ & $8,54 \%$ & $0,00 \%$ & $0,00 \%$ & $9,76 \%$ \\
\hline & PATAGONIA & $2,44 \%$ & $4,88 \%$ & $1,22 \%$ & $0,00 \%$ & $8,54 \%$ \\
\hline & LOS LAGOS & $0,00 \%$ & $3,66 \%$ & $0,00 \%$ & $0,00 \%$ & $3,66 \%$ \\
\hline & $\begin{array}{l}\text { BERNARDO } \\
\text { OHIGGINS }\end{array}$ & $0,00 \%$ & $1,22 \%$ & $0,00 \%$ & $0,00 \%$ & $1,22 \%$ \\
\hline & VALPARAISO & $0,00 \%$ & $1,22 \%$ & $0,00 \%$ & $0,00 \%$ & $1,22 \%$ \\
\hline \multicolumn{6}{|c|}{ Total } & $100 \%$ \\
\hline
\end{tabular}

Tabla 1. Distribución etaria por región según origen de pu chillkatufe

\subsubsection{Región de procedencia y nacionalidad de la población}

Frente al panorama de sentido de pertenencia e identidad, existe un claro reconocimiento a la idea de nación mapuche, donde un $81,48 \%$ de la población participante en estos espacios de aprendizaje reconoce tener nacionalidad mapuche; mientras que un $6,17 \%$ se identifica con otra y un $12,35 \%$ no se identifica con ninguna nacionalidad.

Así también existe prevalencia de estas respuestas en determinadas regiones, siendo la Región de la Araucanía $(29,63 \%)$ la que tiene mayor representatividad, seguida por la Región de Los Ríos (19,75\%) y la Región del Bío-Bío (14,81\%). 


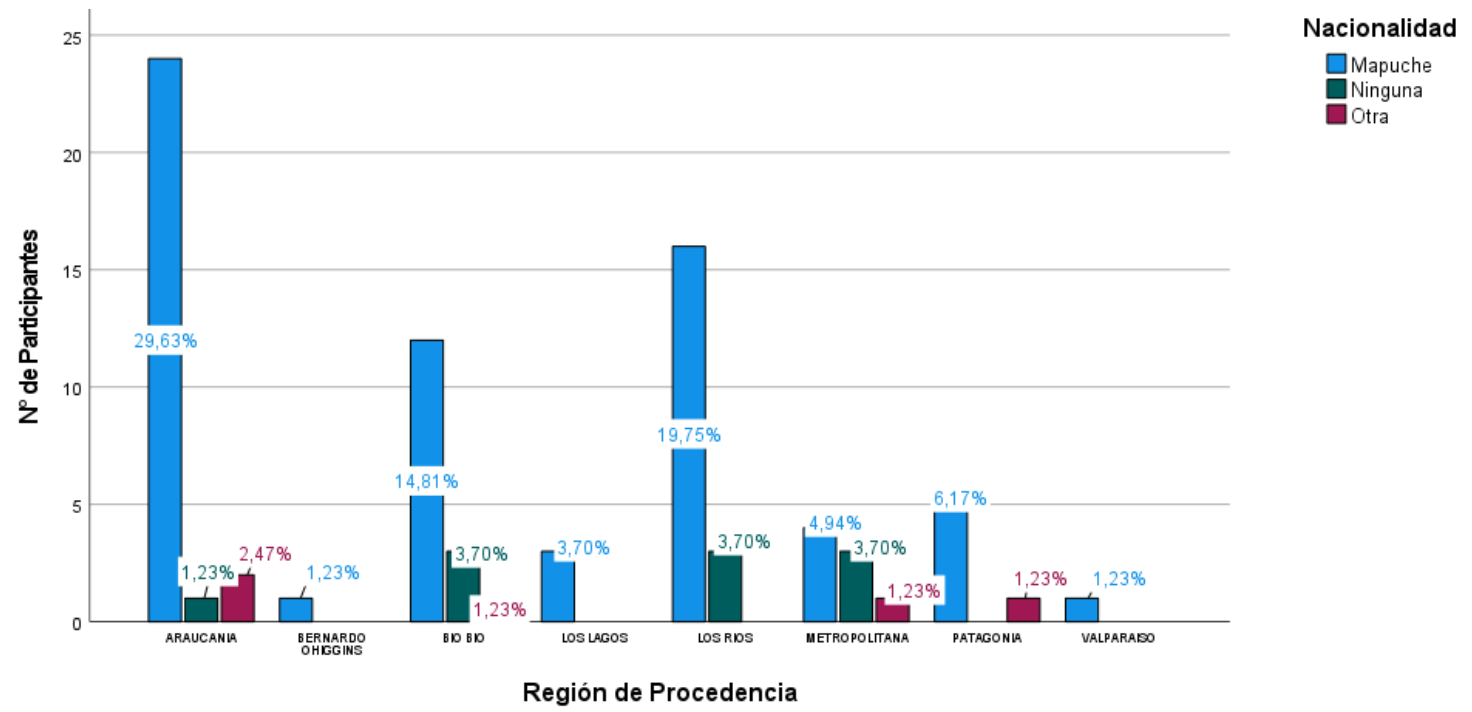

Figura 6. Distribución porcentual según región y nacionalidad de pu chillkatufe

Es posible identificar en estos resultados que la procedencia de las personas que participan de koneltun provienen de las tres regiones preponderantes de migración mapuche, además de ser coherente con los índices de vitalidad que indica la literatura respecto a las regiones donde la población joven no maneja el idioma (Wittig 194).

Igualmente, se observa que la gran parte de las personas que participan de koneltun se autoidentifican como mapuche. Esto reafirma lo identificado en la literatura respecto a la vinculación entre el aprendizaje del idioma y el sentido de pertenencia identitaria, sobre todo si se considera el porcentaje de población mapuche en relación con el total de la población en las regiones de donde procede la mayoría de chillkatufe, como se observa en la Figura 7. 
Artículo. María-Jesús Inostroza, Ester Quero, Aldo Berríos. "Perfil de los participantes de internados de mapuzugun de la organización Mapuzuguletuaiñ๊"

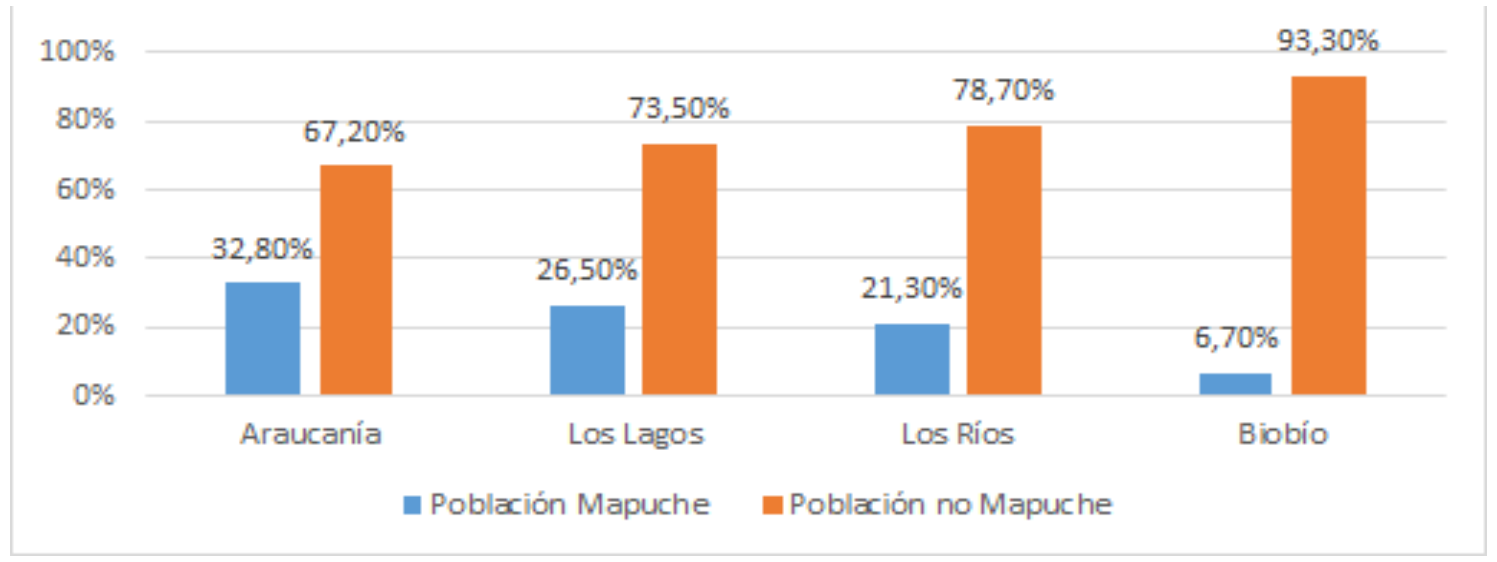

Figura 7. Representatividad de población según Región ${ }^{6}$

Asimismo, si bien los koneltun no se desarrollaron en la región de la Araucanía, la mayoría de los participantes provienen de dicha región. El desplazamiento de chillkatufe fuera de su territorio de residencia puede ser evidencia de la conceptualización del Wallmapu y del mapuzugun como idioma de este territorio. Esto refuerza la idea de una estrategia de revitalización dentro de un contexto territorial delimitado (Naqill 145) y concuerda con el eslogan de Mapuzuguletuaiñ surgido como parte del proceso de oficialización, Mapuzugun mew kiñewkületuaiñ 7 .

\subsection{Trayectoria de participación en instancias de aprendizaje del mapuzugun como segunda lengua antes del koneltun.}

En la actualidad, como ya se indicó, existen diversos espacios de educación formales y no formales que realizan cursos e internados linguiísticos de mapuzugun (Mayo y Castillo 15, Loncón et al. 199).

Según lo evidenciado en esta muestra, el 68,35\% de la población reconoce haber participado en algún curso de mapuzugun, mientras que el 31,65\% no ha participado previamente de dichas instancias. Al consultar sobre las organizaciones en las que han participado con anterioridad, se encuentran instancias de participación formales e informales de aprendizaje, reconociendo mayoritariamente cursos de mapuzugun desarrollados por

\footnotetext{
${ }^{6}$ Elaboración propia, datos adaptados del Ministerio de Desarrollo Social según Casen 2017, “Composición de la población indígena según pueblo por región” y "Porcentaje de la población perteneciente a pueblos indígenas por región".

7 Traducido al castellano como "Volveremos a estar unidos en el mapuzugun".
} 
Mapuzuguletuaiñ, la Universidad Católica de Temuco y la Universidad de la Frontera. En el resto de los cursos desarrollados, no se mencionan organizaciones con repitencia significativa. En relación a lugares e instituciones u organizaciones sociales donde realizaron cursos, se mencionaron 21, siendo Mapuzuguletuaiñ la única organización que abarca cursos en distintos sectores de Wallmapu ${ }^{8}$.

Entre las organizaciones e instituciones que realizan cursos de mapuzugun se hace mención a las siguientes: 1) Mapuzuguletuaiñ (Wallmapu), 2) Universidad de la Frontera, 3) Universidad Catolica de Temuco, 4) Oficina de Asuntos Indígenas (Maipú), 5) Centro Cultural Mapuche (Argentina), 6) Universidad de Concepción, 7) Kom kim Mapudunguaiñ (Santiago), 8) Biblioredes, 9) Re keche (Santiago), 10) Txalkipulli (Pagi), 11) Txafkintuwe (Villarrica), 12) Comunidad Rekawenu (Maullín), 13) Hogar Pelontuwe (Temuco), 14) Departamento de Educacion (Cañete), 15) Coordinadora Penko Lafken (Concepción), 16) Matxümküley (Argentina), 17) Neepin (Concepción), 18) Universidad de los Lagos, 19) Conadi (Santiago), 20) Universidad de Chile y 21) Departamento de Cultura (Rancagua).

Respecto a la participación en internados lingüísticos, el 53,66\% de los encuestados declaró haber participado en estos, mientras que un 46,34\% informó que no había acudido a algún koneltun, arrojando una prevalencia de participación mayoritaria en la región de la Araucanía a través de Mapuzuguletuaiñ. Por otro lado, existe un alto índice de pu chillkatufe participante en distintos internados, los que dan cuenta de que existe un interés en el aprendizaje del idioma y que esto se sustenta a través del tiempo.

Entre las organizaciones e instituciones que realizan koneltun se mencionaron de las siguientes: 1) Mapuzuguletuaiñ ${ }^{9}$, 2) FEMAE (Federación Mapuche de Estudiantes), 3) Universidad de Concepción, 4) Püchikeche Choike y 5) Inche Willimapu.

\section{Conclusiones}

\footnotetext{
${ }^{8}$ Angol, Pucón, Padre las Casas, Temuco, Lanalhue, Valdivia y Concepción.

${ }^{9}$ Organización con mayor nivel de recurrencia en la mención de las respuestas y la que abarca mayores sectores de Wallmapu: Angol, Temuco, Coñaripe,Victoria, Galvarino, Victoria, Curarrehue, Carahue, Concepción, Tirúa, Quidico, Valdivia, San Carlos de Bariloche, Püchilewfu, Osorno y Rancagua.
} 
Artículo. María-Jesús Inostroza, Ester Quero, Aldo Berríos. "Perfil de los participantes de internados de mapuzugun de la organización Mapuzuguletuaiñ”

La revisión de antecedentes expuestos busca ser una contribución a la revitalización del Mapuzugun mediante las acciones desarrolladas por el Instituto Nacional de la Lengua Mapuche Mapuzuguletuaiñ. En esta investigación se ha respondido a los objetivos planteados referentes a dar a conocer el perfil demográfico y la trayectoria participativa de aprendizaje de quienes asisten a estos espacios de educación no formal y comunitaria bajo el método de inmersión lingüística en los koneltun de mapuzugun.

Los resultados muestran como perfil demográfico una tendencia a la participación de adultos (entre 20 y 25 años) con estudios universitarios completos. Este perfil es coherente con los integrantes de la organización y los espacios donde desarrolla algunas de sus actividades. Se observa además que la mayoría de los y las participantes se auto-identifican como mapuche, lo que es congruente con sus territorios de procedencia, donde existe mayor concentración de población mapuche (Araucanía, Bío-Bío, Los Lagos). Igualmente, el desplazamiento de pu chillkatufe fuera de su región de origen para asistir a los koneltun, reafirma la idea de que el Wallmapu es el territorio de una nación y que el mapuzugun es el idioma de dicho territorio.

En términos de los resultados, se puede concluir además que existe un aprendiz con una trayectoria de participación constante y reiterativa, la que se concreta dentro de una misma organización. Asimismo, se puede señalar que la estrategia de revitalización de Mapuzuguletuaiñ tiene una focalización a largo plazo, a diferencia de otras instituciones y/u organizaciones. Esto se ve reflejado en la orientación metodológica elegida por este txokiñche, denominada Enfoque Comunicativo, que se sustenta en el desarrollo sistemático y progresivo en el tiempo de las competencias linguiísticas. Es así como su organización en niveles involucra un número de horas necesarias e ineludibles dedicadas al estudio.

Entre las contribuciones, el estudio visibiliza la situación del mapuzugun y su estatus como lengua oprimida, siendo los internados lingüísticos espacios de aprendizaje sustentables en el tiempo. Además, muestra los primeros acercamientos a la descripción de personas interesadas en preservar, mantener y reproducir la lengua mapuche. Otro de los elementos de relevancia es que el presente estudio permite observar que las acciones desarrolladas por Mapuzuguletuaiñ, la identifican como una organización que trabaja en pos de la revitalización del mapuzugun, reconociendo la estrategia de enseñanza-aprendizaje 
como una acción a largo plazo que ha sido identificada en la literatura y por quienes han sido participantes de koneltun.

Las condiciones contextuales (accesibilidad geográfica y escasez de personal técnico en logística) permitieron aplicar el instrumento en solo dos instancias, por lo que se debe considerar que estos resultados no son necesariamente representativos o generalizables a todos los koneltun realizados durante la temporada.

Finalmente, estos resultados se pueden proyectar a un estudio longitudinal del perfil que incorpore el nivel de logro de los resultados de aprendizaje respecto a las instancias de participación de pu chillkatufe. Igualmente, sería interesante explorar las motivaciones y creencias de este grupo de personas respecto a las instancias de koneltun en general. De la misma manera, y dado el alto número de participantes con trasfondo profesional, queda por indagar la relación de los txokiñche con las instituciones académicas a través de la realización de cursos, perfeccionamientos o programas de extensión.

\section{REFERENCIAS}

Adelaar, Willem. The languages of the Andes. Cambridge: Cambridge University Press, 2004. Impreso.

Ajello, Anna María. "La motivación para aprender". Manual de psicología de la educación Coord. Clotilde Pontecorvo. Madrid: Editorial Popular, 2003. 251-271. Impreso.

Amberga, Jerónimo de. "Estudio y enseñanza del Mapuche en la era colonial". Revista Chilena de Historia y Geografía 11.15 (1914): 420-25. Impreso.

Antimil, Jaime, y Aldo Olate. "El escenario actual de la lengua mapuche en un territorio. Estudio de caso desde la historia y la sociolingüística". Nueva Revista Del Pacífico 72 (2020): 116-43. Impreso.

Arnstein, Sherry "A Ladder of Citizen Participation”. Journal of the American Planning Association 85.1 (2019): 24-34.

Becerra Parra, Rodrigo. "Folil Mapudungun: organización de un método con enfoque comunicativo para la enseñanza-aprendizaje de la lengua Mapuche". Revista Alpha 46 (2018): 89-110.

Brown, H. Douglas y Heekyeong Lee. Teaching by Principles: An Interactive Approach to Language Pedagogy. Londres: Pearson Education, 2015. Impreso.

Canales Tapia, Pedro. "Escuelas chilenas en contextos mapuche. Integración y resistencia, 1860-1950". Última Década 6.9 (1998): 3-18. Impreso.

Catrileo, María. La lengua mapuche en el siglo XXI. Valdivia: Universidad Austral de Chile, 2010. Impreso.

Cisternas, César. "Educación intercultural bilingüe en La Araucanía (Chile): Entre la inclusión y el nacionalismo minoritario". Actualidades Investigativas en Educación 18.2 (2018): 2-25. 
Artículo. María-Jesús Inostroza, Ester Quero, Aldo Berríos. "Perfil de los participantes de internados de mapuzugun de la organización Mapuzuguletuaiñ"

Cisternas, César, and Arturo Vallejos-Romero. "La planificación lingüística desde una sociología sistémica del lenguaje: Un análisis desde los casos del mapudungún, el sami y el maorí”. RLA. Revista de Lingüística Teórica y Aplicada 57.2 (2019): 11736.

CONADI. Azümchefe. Grafemario único del idioma mapuche. Estudio para la definición de un grafemario para la lengua mapuche. Temuco: Departamento de Cultura y Educación, CONADI, 2008. Impreso.

Hernández, Arturo, Nelly Ramos y Rosa Huenchulaf. Gramática básica de la lengua mapuche. Temuco: Editorial UC, 2011. Impreso.

Garabide. "Nuestro trabajo." Garabide, https://www.garabide.eus/espanol/garabide/nuestrotrabajo/ s. p. Web. 27 febr, 2021.

Gardner, Robert. "Integrative Motivation and Second Language Acquisition". Motivation and Second Language Acquisition. Ed. Zoltan Dörnyei y Richard Schmidt. Honolulu: University of Hawaii, 2001. 1-19. Impreso.

Gardner, Robert. Social Phsychology and Second Language Learning: The Role of Attitude and Motivation. Londres: Edward Arnold, 1985. Impreso.

Gaune, Rafael. "El jesuita como traductor. Organización, circulación y dinámicas de la Compañía de Jesús en Santiago de Chile, 1593-1598." Historia Crítica 50 (2013): 13-35.

Gundermann, Hans, Jaqueline Canihuan, Alejandro Clavería y César Faúndez. "El mapuzugun, una lengua en retroceso". Atenea 503 (2011): 111-131.

Gundermann, Hans, Jaqueline Canihuan, Alejandro Clavería y César Faúndez. "Permanencia y desplazamiento, hipótesis acerca de la vitalidad del mapuzugun”. RLA, Revista de Lingüística Teórica y Aplicada, 47.1 (2009): 37-60.

Mapuzuguletuaiñ. "Mapuzuguletuaiñ Wallmapu mew". Mapuzuguletuaiñ, www.mapuzuguletuain.cl/index.php/acerca-de/ s.p. Web. 21 Mar. 2021.

Lagos, Cristián. "El mapudungún en Santiago de Chile: Vitalidad y representaciones sociales en los mapuches urbanos". RLA. Revista de Lingüística Teórica y Aplicada 50.1 (2012): 161-84.

Loncon, Elisa. "Mapuzugun mapuce rakizuwam mew: Cumgefuy, cumlen ka ñi cumleam". Americania 0 (2017): 208-223.

Loncon, Elisa. "El mapuzugun desde el pensamiento mapuche: Pasado, presente y futuro". Americania 0 (2017): 204-209.

Loncon, Elisa y Silvia Castillo. "Neologismos en mapuzugun: palabras creadas en un proceso de enseñanza y de aprendizaje”. Literatura y Lingüística 38 (2018): 195-212. Impreso.

Llanquinao, Gabriel, Gastón Salamanca y Fernando Teillier. "Aprendizaje del mapunzugun desde metodologías propias: perspectivas y avances para la revitalización de lenguas originarias". Revista Historia de la Educación Latinoamericana 21.33 (2019): 12143.

Mayo, Simona, y Silvia Castillo. "Movimientos autónomos por la lengua mapuche en Wallmapu”. LASA Forum 50.1 (2019): 15-18.

McIvor, Onowa. "Indigenous Language Revitalization and Applied Linguistics: Parallel Histories, Shared Futures?”. Annual Review of Applied Linguistics 40 (2020): 7896

Moore, Sylvia. "Language and Identity in an Indigenous Teacher Education Program". International Journal of Circumpolar Health 78.2 (2019). 
Moreno, Karen, Juan Enrique Bostelmann, Cintia Macías, Ximena Navarro-Harris, Ricardo De Pol-Holz y Mario Pino. "A late pleistocene human footprint from the pilauco archaeological site, Northern Patagonia, Chile”. PLoS ONE 14.4 (2019).

Moseley, Christopher. Atlas de las lenguas del mundo en peligro. París: Ediciones UNESCO, 2010.

Moulian, Rodrigo, María Catrileo, Jaqueline Caniguan y Felipe Hasler. "Consonancias léxicas andinas en el lenguaje ritual mapuche williche: relaciones translingüísticas y estratificación histórica." Estudios Filológicos 64 (2019): 251-274. Impreso.

Naguil, Víctor. "Entre comunitarismo y nacionalismo: El caso mapuche, 1990-2010". RIPS: Revista de Investigaciones Políticas y Sociológicas 12.1 (2013): 39-69.

Namuncura, Alina. "El mapuzugun en el aula: el informe de una experiencia". Revista de Lenguas y Literatura Indoamericanas 20 (2018): 56-67.

Naqill, Viktor. "Lengua y territorio: relación estratégica para la revitalización del mapuzugun". Caracol 20 (2020): 134-165

Nicholas, Sheilah. "Becoming 'fully' Hopi: The role of Hopi language in the contemporary lives of Hopi youth - A Hopi case study of language shift and vitality". Tesis de $\mathrm{PhD}$, Universidad de Arizona, 2008.

Ñanculef, Ana y Cristian Cayupán. Kuifike Zugu: Discursos, relatos y oraciones rituales en mapuzugun. Temuco: Comarca Ediciones, 2016. Impreso.

Olate, Aldo, Gerardo Chandía, Jacqueline Caniguan y Diego Lizarralde. "Academias de la lengua mapuche en Chile: un espacio para la revitalización lingüística”. Quo Vadis, Romania 53-54 (2019): 42-57.

Ovalle, Alonso de. Histórica relación del Reino de Chile y de las misiones y ministerios que ejercita en él la Compañía de Jesús... Roma: Francisco Caballo. 1646. Impreso.

Pinto, Jorge. "El parlamento de Coz Coz y la marcación de Painemal, un giro en la relación de la sociedad chilena con el pueblo mapuche, 1907-1913." Tefros 10.1-2 (2012): $1-23$.

Salas, Adalberto. "Hablar en mapuche es vivir en mapuche. Especificidad de la relación lengua/cultura”. Revista de Lingüística Teórica y Aplicada 25 (1987): 27-35.

Stake, Robert. Investigación con estudio de casos. Madrid: Morata. 2007.

Teillier, Fernando. "Vitalidad lingüística del mapudungun en chile y epistemología del hablante." RLA. Revista de Lingüística Teórica y Aplicada 51.1 (2013): 53-70.

Tomás, María, Malena Pell, Hermelinda Tripailafken y Paula Cecchi. "Del proyecto al compromiso: interculturalidad y agencias entre colectivos mapuche y políticas públicas en extensión universitaria”. E+E: Estudios de Extensión y Humanidades 7.10 (2020): $33-49$

Wittig, Fernando e Israel Farías. "Trayectorias sociolingüísticas intergeneracionales. Una categoría para el análisis de procesos de transmisión y desplazamiento del mapuzugun”. Atenea (Concepción) 517 (2018): 123-138.

Wittig, Fernando. "Adquisición y transmisión del mapudungún en hablantes urbanos." Literatura y Linguistica 23 (2011): 193-211.

Zuñiga, Fernando. "Mapudunguwelaymi am? ‘¿Acaso ya no hablas mapudungun?' Acerca del estado actual de la lengua mapuche". Estudios Públicos 105 (2007): 9-24. Impreso. 
Artículo. María-Jesús Inostroza, Ester Quero, Aldo Berríos. "Perfil de los participantes de internados de mapuzugun de la organización Mapuzuguletuaiñ”

Zúñiga, Fernando y Aldo Olate. "El estado de la lengua mapuche, diez años después". El pueblo mapuche en el siglo XXI. Ed. Isabel Aninat, Verónica Figueroa y Ricardo González. 2017. 343-74. 